\title{
Modeling and analysis of the symptomatic and asymptomatic infections of swine flu with optimal control
}

\author{
Akhil Kumar Srivastav' ${ }^{1} \operatorname{Mini}_{\operatorname{Ghosh}^{1}}$ (D)
}

Received: 2 September 2016/Accepted: 19 September 2016/Published online: 6 October 2016

(C) Springer International Publishing Switzerland 2016

\begin{abstract}
Swine flu is an infectious disease which spreads very rapidly in the population. Infected droplets are expelled into the air by swine flu infected individuals through coughing and sneezing. This disease is transmitted to susceptible individuals by inhalation or ingestion of these infected droplets containing virus. In this paper, we propose and analyze a mathematical model for Swine Flu by considering symptomatic and asymptomatic infections. It is assumed that the transmission rates due to symptomatic and asymptomatic individuals are different. The mathematical model is formulated by assuming simple mass-action type incidence. The basic reproduction number $R_{0}$ of the model is computed and the local and the global stabilities of different equilibria of the model are studied. Further, this model is extended to optimal control model. The optimal control model is analyzed using Pontryagin's Maximum Principle and is solved numerically using MATLAB. Finally numerical simulation is performed to see the effect of optimal control on the infected population. It is observed that optimal control model gives better result compared to the model without optimal control as it reduces the number of infectives significantly in a desired interval of time.
\end{abstract}

Mini Ghosh

minighosh@vit.ac.in

Akhil Kumar Srivastav

akil.ksrivastav2014@vit.ac.in

1 Division of Mathematics, School of Advanced Sciences, VIT University Chennai Campus, Vandalur-Kelambakkam Road, Chennai 600 127, India
Keywords Swine influenza - Mathematical model · Stability analysis - Optimal control

\section{Introduction}

In 21 st century, Swine Influenza has emerged as a deadly infectious disease. There are mainly three types of influenza, namely, Influenza A, Influenza B and Influenza C. Influenza of type $A$ and type $B$ are more prevalent in human population and are of particular public health concern. Influenza A have many subtype strains, e.g. H1N1, H2N2, H3N2, etc. Here we are studying H1N1 subtype which is currently circulating in human population and is known as Swine Flu.

Many Influenza outbreaks are reported in all over the world e.g. 1918 pandemic, 1976 US outbreak, 1988 US outbreak, 2007 Philippine outbreak, 2009 Northern Ireland outbreak, 2015 India outbreak.

In 1918, a new disease was identified in pig, that is known as swine influenza. In human, this pandemic is associated with the infection of $\mathrm{H} 1 \mathrm{~N} 1$ virus. This virus also circulates in pig, so this pandemic was called zoonosis (from swine to humans, or from humans to swine). In this pandemic near about 500 million people were infected and 50-100 million people were killed across the world. We can say that it was one of the deadliest disasters in human history.

Recently, in 2015, an outbreak of swine flu (H1N1 virus) had been reported in India, which was similar to 2009 Influenza pandemic. This outbreaks were reported in late 2014 and early 2015. In March 2015, Health Ministry released a data and according to this data 31,974 people had been reported infected and 1895 person had died. The largest number of cases and deaths due to the disease occurred in states like Delhi, Madhya Pradesh, Rajasthan, and Gujarat in India (MHFW 2016). 
Symptoms of swine flu are as follows:

In adults: difficulty in breathing, pain or pressure in the chest, sudden dizziness, confusion, severe vomiting, low temperature.

In children: fast breathing or working hard to breathe, bluish skin color, not drinking enough fluids, not waking up or not interacting, being so irritable that the child does not want to be held, flu-like symptoms which improve but then return with fever and worse cough, fever with a rash, being unable to eat, having no tears when crying (CDC 2016).

There are several mathematical models to describe and analyze the transmission dynamics of influenza. In Sharomi et al. (2011), authors presented a deterministic model for the transmission dynamics of swine influenza (H1N1) in the presence of an imperfect vaccine and use of drug therapy. Their analysis reveals that if the associated reproduction number is less than unity then the system exhibits vaccine induced backward bifurcation. Mathematical models for AH1N1/09 incorporating spatio-temporal elements are studied in Gonzlez-Parra et al. (2011). Authors validated their model with the time series notifications from selected regions. In Changpuek et al. (2013), authors considered a mathematical model for swine flu by dividing the whole population into different age groups and assuming different rates of transmission in different age groups. Optimal control theory is applied to a mathematical model for swine flu in Aldila et al. (2014) where it is identified that medical mask intervention can reduce the disease prevalence significantly. Our work is based on Pongsumpun and Tang (2011), where authors have analyzed a mathematical model for swine flu by considering both symptomatic (with symptoms) and asymptomatic (without symptoms) infections. Here total population was considered as constant and disease transmission was assumed to follow standard incidence. This study is suitable for shorter span of time as population need not be a constant over a longer span of time. As swine flu is endemic now so study of long term dynamics of disease is required and total population should be variable. Hence we have formulated a mathematical model for swine flu by considering total population variable. Additionally we considered simple mass action type incidence for disease transmission as transmission of this disease is very much dependent on population size. The rates of movement from symptomatic and asymptomatic class to class of quarantine individuals are taken different in our model where as in Pongsumpun and Tang (2011) they were same.

Later our proposed model is extended to optimal control problem to get the optimal control profile related to rate of transmission and rate of quarantine.

The remaining of this paper is organized as follows: Sect. 2 describes the basic model and corresponding basic reproduction number; Sect. 3 presents the equilibria and their stability; Sect. 4 discusses the numerical simulation and results of the proposed model; Sect. 5 discusses the optimal control model and its analysis; Sect. 6 demonstrates the numerical simulation results of the optimal control model and finally Sect. 7 concludes the paper.

\section{Mathematical model}

We first divide the total population $N(t)$ into six compartments, namely, susceptible individuals (S), exposed individuals (E), symptomatic infective individuals $\left(I_{s}\right)$, asymptomatic infective individuals $\left(I_{a}\right)$, quarantine individuals $(Q)$, recovered (R). Hence $N=S+E+$ $I_{s}+I_{a}+Q+R$. It is assumed that the total population is varying and homogeneously mixed i.e., all people are equally likely to be infected by the infectious individuals if they come into contact. It is assumed that susceptible individuals after being exposed to the infection can move to any one of the following infective classes, namely, symptomatic infective, asymptotic infective with different transmission rates. Both types of infectious individuals can move to class of quarantine individuals. Quarantine individuals may recover and after recovery it move to recovered class. However the rates of recovery may vary from one compartment to another. Keeping the above facts/assumptions in mind, a mathematical model is proposed as follows:

$\frac{\mathrm{d} S}{\mathrm{~d} t}=\Lambda-\mu S-\gamma I S$

$\frac{\mathrm{d} E}{\mathrm{~d} t}=\gamma I S-\left(\beta_{s}+\beta_{a}\right) b E-\mu E$

$\frac{\mathrm{dI}_{\mathrm{s}}}{\mathrm{d} t}=\beta_{s} b E-\delta I_{s}-\mu I_{s}-\eta I_{s}$

$\frac{\mathrm{dI}_{\mathrm{a}}}{\mathrm{d} t}=\beta_{a} b E-v I_{a}-\mu I_{a}$

$\frac{\mathrm{dQ}}{\mathrm{d} t}=\delta I_{s}+v I_{a}-\mu Q-\psi Q$

$\frac{\mathrm{dR}}{\mathrm{d} t}=\psi Q-\mu R$.

The flow diagram of the proposed model is shown in Fig. 1.

Let $\left(S, E, I_{s}, I_{a}, Q, R\right)$ be any solution with positive initial condition. Then we have

$N=S+E+I_{a}+I_{s}+Q+R$.

Then the time derivative of the total population $N(t)$ is given by

$\frac{\mathrm{d} N}{\mathrm{~d} t}=\Lambda-\mu N-\eta I_{s}$,

which shows that $\frac{\mathrm{d} N}{\mathrm{~d} t}$ is bounded by $\Lambda-\mu N$. Now using standard comparison theorem (Lakshmikantham et al. 
Table 1 Description of parameters

\begin{tabular}{ll}
\hline Parameter & Description \\
\hline$\mu$ & Recruitment rate \\
$\mu$ & Natural death rate \\
$\gamma$ & Transmission rate of swine flu \\
$1 / b$ & Incubation period of swine flu in human \\
$\beta_{s}$ & Transmission probability of swine flu to the human so \\
& that person becomes symptomatic patient \\
$\beta_{a}$ & Transmission probability of swine flu to the human so \\
& that person becomes asymptomatic patient \\
$v$ & Rate of quarantine for symptomatic infectious person \\
$\psi$ & Rate of quarantine for asymptotic infectious person \\
& Rate of movement of individuals from quarantine class to \\
$u_{1}$ & recovered class \\
$u_{2}$ & Control parameter on the transmission rate \\
& Control parameter on the progression of symptomatic \\
$\eta$ & individuals to quarantine class
\end{tabular}

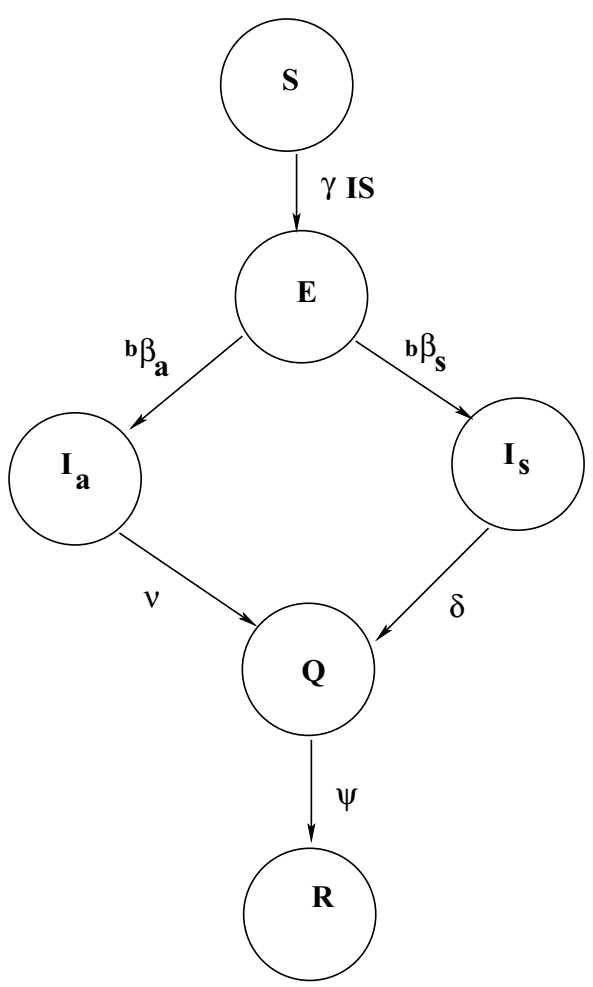

Fig. 1 Flow diagram of the model

1989), it is easy to observe that $N(t) \leq \frac{\Lambda}{\mu}$ if $N(0) \leq \frac{\Lambda}{\mu}$. Hence the region $\Omega=\left\{\left(S, E, I_{a}, I_{s}, Q, R\right): S+E+I_{a}+\right.$ $\left.I_{s}+Q+R \leq \frac{\Lambda}{\mu}\right\}$ is positively invariant.
The basic reproduction number $R_{0}$

The system (1) has the disease-free equilibrium (DFE) $E_{0}$ as

$$
E_{0}=\left(S^{0}, E^{0}, I_{a}{ }^{0}, I_{s}{ }^{0}, Q^{0}, R^{0}\right)=\left(\frac{\Lambda}{\mu}, 0,0,0,0,0\right)
$$

To find the basic reproduction number $R_{0}$, we follow the same method as discussed in Korobeinikov and Wake (2002), Li and Muldowney (1995) and using the same notations, the matrices $\mathrm{F}$ and $\mathrm{V}$, for the new infection terms and the remaining transfer terms respectively, corresponding to the system (1) are computed as follows:

$\mathcal{F}=\left(\begin{array}{c}\gamma I S \\ 0 \\ 0\end{array}\right)=\left(\begin{array}{c}\gamma\left(I_{a}+I_{s}\right) S \\ 0 \\ 0\end{array}\right)$

and

$\mathcal{V}=\left(\begin{array}{c}\beta_{s} b E+\beta_{a} b E+\mu E \\ -\beta_{s} b E+\mu I_{s}+\delta I_{s}+\eta I_{s} \\ -\beta_{a} b E+\mu I_{a}+v I_{a}\end{array}\right)$

We can find $F$ and $V$ as follows: $\mathrm{F}=$ Jacobian of $\mathcal{F}$ at $E_{0}=$

$F=\left(\begin{array}{ccc}0 & \gamma S^{0} & \gamma S^{0} \\ 0 & 0 & 0 \\ 0 & 0 & 0\end{array}\right)$

and $\mathrm{V}=$ Jacobian of $(\mathcal{V})$ at $E_{0}=$

$V=\left(\begin{array}{ccc}b\left(\beta_{s}+\beta_{a}\right)+\mu & 0 & 0 \\ -b \beta_{s} & \mu+\delta+\eta & 0 \\ -b \beta_{a} & 0 & \mu+v\end{array}\right)$

and it follows that

$F V^{-1}=\left(\begin{array}{ccc}m_{11} & m_{12} & m_{13} \\ 0 & 0 & 0 \\ 0 & 0 & 0\end{array}\right)$

where

$m_{11}=\frac{\gamma b S^{0}}{b \beta_{s}+b \beta_{a}+\mu}\left(\frac{\beta_{s}}{\mu+\delta+\eta}+\frac{\beta_{a}}{\mu+v}\right)$,

$m_{12}=\frac{\gamma S^{0}}{\mu+\delta}, m_{13}=\frac{\gamma S^{0}}{\mu+v}$.

Then the basic reproduction number $R_{0}$ is given by the largest eigenvalue of $F V^{-1}$ and is obtained as follows:

$R_{0}=\frac{\gamma b S^{0}}{b \beta_{s}+b \beta_{a}+\mu}\left(\frac{\beta_{s}}{\mu+\delta+\eta}+\frac{\beta_{a}}{\mu+v}\right)$

The reproduction number $R_{0}$ gives the average number of infected humans generated by one infected human in a fully susceptible population in his/her whole infectious period. 


\section{Equilibria and their stability}

In addition to the disease-free equilibrium $E_{0}$, the model system (1) also has a unique endemic equilibrium point $E_{1}=\left(S^{*}, E^{*}, I_{a}^{*}, I_{s}^{*}, Q^{*}, R^{*}\right)$, where

$$
\begin{aligned}
& S^{*}=\frac{\left(\beta_{s}+\beta_{a}\right) b+\mu}{\gamma\left(d_{1}+d_{2}\right)}, E^{*}=\frac{\frac{\mu}{\gamma}\left(R_{0}-1\right)}{\left(d_{1}+d_{2}\right)}, I_{s}^{*}=d_{1} E^{*}, I_{a}{ }^{*}=d_{2} E^{*}, \\
& Q^{*}=\frac{\delta d_{1}+v d_{2}}{\mu+\psi} E^{*}, R^{*}=\frac{\psi Q^{*}}{\mu}
\end{aligned}
$$

and

$$
d_{1}=\frac{\beta_{s} b}{\mu+\delta+\eta}, d_{2}=\frac{\beta_{a} b}{\mu+v} .
$$

From above expressions it is clear that $E^{*}$ is positive only when $R_{0}>1$. Hence the endemic equilibrium point $E_{1}$ exists whenever $R_{0}>1$.

\section{Global stability of disease free equilibrium (DFE)}

To prove the global stability of disease free equilibrium, we are using the theorem by Castillo-Chavez et al. ( $\mathrm{Li}$ and Muldowney 1996).

Theorem 1 If the given mathematical model can be written in the form:

$\frac{d X}{d t}=F(X, Y)$, and $\frac{d Y}{d t}=G(X, Y), G(X, 0)=0(*)$

where $X=S, Y=\left(E, I_{a}, I_{s}\right)^{T}$, denoting the classes of uninfected and flu infected individuals respectively.

The DFE is represented here by $E_{0}=\left(X_{0}, 0\right)=\left(\frac{\Lambda}{\mu}, 0\right)$.

For the global asymptotic stability of $E_{0}$, the condition $\left(H_{1}\right)$ and $\left(H_{2}\right)$ given below must be satisfied.

$H_{1}$ : for $\frac{d X}{d t}=F\left(X_{0}, 0\right), X_{0}$ is global asymptotically stable, $H_{2}: G(X, Y)=A Y-\widehat{G}(X, Y), \widehat{G}(X, Y) \geq 0$ here $A$ $=D_{Y} G\left(X_{0}, 0\right)$ is M- matrix (In M-matrix, all the off diagonal element of matrix are non-negative). If the given system of differential equation in mathematical model satisfies the given condition in $(*)$ then the point $E_{0}=$ $\left(X_{0}, 0\right)$ is a global asymptotically stable equilibrium of given mathematical model provided $R_{0}<1$. And for the given mathematical model, the result is shown in the next theorem, as given below.

Theorem 2 The equilibrium point $E_{0}=\left(X_{0}, 0\right)$ of the system (1) is global asymptotically stable (G.A.S.) provided $R_{0}<1$ and the conditions given in $(*)$ are satisfied.
Proof By using Theorem 1 to our model system (1), we get

$F\left(X_{0}, 0\right)=\Lambda-\mu S, G(X, Y)=A Y-\widehat{G}(X, Y)$

where

$A=\left(\begin{array}{ccc}-\left[b\left(\beta_{s}+\beta_{a}\right)+\mu\right] & \gamma S^{0} & \gamma S^{0} \\ b \beta_{s} & -(\mu+\delta+\eta) & 0 \\ b \beta_{a} & 0 & -(\mu+v)\end{array}\right)$

and

$\hat{G}(X, Y)=\left(\begin{array}{c}\hat{G}_{1}(X, Y) \\ \hat{G}_{2}(X, Y) \\ \hat{G}_{3}(X, Y)\end{array}\right)=\left(\begin{array}{c}\gamma\left(I_{a}+I_{s}\right)\left(S^{0}-S\right) \\ 0 \\ 0\end{array}\right)$.

Here we can easily see $S^{0} \geq S$, hence $\widehat{G}(X, Y) \geq 0$ for all $(\mathrm{X}, \mathrm{Y})$, we can also notice that the matrix $\mathrm{A}$ is M matrix (by definition of M matrix) .

Hence the DFE $\left(E_{0}\right)$ is globally stable.

Theorem 3 The endemic equilibrium $E_{1}=$ $\left(S^{*}, E^{*}, I_{s}{ }^{*}, I_{a}{ }^{*}, Q^{*}, R^{*}\right)$ of the given mathematical model is globally asymptotically stable.

Proof For the global stability result, we will use the method discussed in Korobeinikov and Wake (2002), Li and Muldowney (1995). Here we consider the following Lyapunov function:

$$
\begin{aligned}
& V_{1}=K_{1}\left(S-S^{*}-S^{*} \ln \frac{S}{S^{*}}\right)+K_{2}\left(E-E^{*}-E^{*} \ln \frac{E}{E^{*}}\right) \\
& +K_{3}\left(I_{s}-I_{s}{ }^{*}-I_{s}{ }^{*} \ln \frac{I_{s}}{I_{s}{ }^{*}}\right)+K_{4}\left(I_{a}-I_{a}{ }^{*}-I_{a}{ }^{*} \ln \frac{I_{a}}{I_{a}{ }^{*}}\right)
\end{aligned}
$$

Then the time derivative of $V_{1}$ is given by

$$
\begin{aligned}
& \frac{\mathrm{d} V_{1}}{\mathrm{~d} t}=K_{1}\left(1-\frac{S^{*}}{S}\right) \frac{\mathrm{d} S}{\mathrm{~d} t}+K_{2}\left(1-\frac{E^{*}}{E}\right) \frac{\mathrm{d} E}{\mathrm{~d} t}+K_{3}\left(1-\frac{I_{s}^{*}}{I_{s}}\right) \\
& \frac{\mathrm{d} I_{s}}{\mathrm{~d} t}+K_{4}\left(1-\frac{I_{a}^{*}}{I_{a}}\right) \frac{\mathrm{d} I_{a}}{\mathrm{~d} t}
\end{aligned}
$$

Now from the mathematical model we put the expressions for $\frac{\mathrm{d} S}{\mathrm{~d} t}, \frac{\mathrm{d} E}{\mathrm{~d} t}, \frac{\mathrm{d} I_{s}}{\mathrm{~d} t}, \frac{\mathrm{d} I_{a}}{\mathrm{~d} t}$ in the above equation, which gives

$$
\begin{aligned}
\frac{\mathrm{d} V_{1}}{\mathrm{~d} t}= & K_{1}\left(1-\frac{S^{*}}{S}\right)[\Lambda-\mu S-\gamma I S]+K_{2}\left(1-\frac{E^{*}}{E}\right) \\
& \times\left[\gamma I S-\left(b \beta_{s}+b \beta_{a}+\mu\right) E\right] \\
& +K_{3}\left(1-\frac{I_{s}^{*}}{I_{s}}\right)\left[b \beta_{s} E-(\delta+\mu+\eta) I_{s}\right] \\
& +K_{4}\left(1-\frac{I_{a}{ }^{*}}{I_{a}}\right)\left[b \beta_{a} E-(v+\mu) I_{a}\right]
\end{aligned}
$$

The mathematical model system satisfies the following relation at the equilibrium point. 


$$
\begin{aligned}
& \Lambda=\mu S^{*}+\gamma I^{*} S^{*},\left[\left(\beta_{s}+\beta_{a}\right) b+\mu\right]=\frac{\gamma I^{*} S^{*}}{E^{*}}, \\
& (\delta+\mu+\eta)=\frac{b \beta_{s} E^{*}}{I_{s}{ }^{*}},(v+\mu)=\frac{b \beta_{a} E^{*}}{I_{a}{ }^{*}} .
\end{aligned}
$$

Putting all the above expressions in (2) we get,

$$
\begin{aligned}
\frac{\mathrm{d} V_{1}}{\mathrm{~d} t} & =K_{1}\left(1-\frac{S^{*}}{S}\right)\left[\mu S^{*}+\gamma I^{*} S^{*}-\mu S-\gamma I S\right] \\
& +K_{2}\left(1-\frac{E^{*}}{E}\right)\left[\gamma I S-\left(\frac{\gamma I^{*} S^{*}}{E^{*}}\right) E\right] \\
& +K_{3}\left(1-\frac{I_{s}^{*}}{I_{s}}\right)\left[b \beta_{s} E-\frac{b \beta_{s} E^{*}}{I_{s}{ }^{*}} I_{s}\right] \\
& +K_{4}\left(1-\frac{I_{a}{ }^{*}}{I_{a}}\right)\left[b \beta_{a} E-\frac{b \beta_{a} E^{*}}{I_{a}{ }^{*}} I_{a}\right]
\end{aligned}
$$

Then

$$
\begin{aligned}
\frac{\mathrm{d} V_{1}}{\mathrm{~d} t}= & -\left(K_{1}\right)\left(\frac{\left(S^{*}-S\right)^{2}}{S}\right) \mu+K_{1}\left(1-\frac{S^{*}}{S}\right)\left[\gamma I^{*} S^{*}-\gamma I S\right] \\
& +K_{2}\left(1-\frac{E^{*}}{E}\right)\left[\gamma I S-\left(\frac{\gamma I^{*} S^{*}}{E^{*}}\right) E\right] \\
& +K_{3}\left(1-\frac{I_{s}^{*}}{I_{s}}\right)\left[b \beta_{s} E-\frac{b \beta_{s} E^{*}}{I_{s}^{*}} I_{s}\right] \\
& +K_{4}\left(1-\frac{I_{a}^{*}}{I_{a}}\right)\left[b \beta_{a} E-\frac{b \beta_{a} E^{*}}{I_{a}^{*}} I_{a}\right] \\
\frac{\mathrm{d} V_{1}}{\mathrm{~d} t}= & \frac{-K_{1} \mu\left(S^{*}-S\right)^{2}}{S}+g\left(x_{1}, x_{2}, x_{3}, x_{4}\right)
\end{aligned}
$$

where

$$
\begin{aligned}
\frac{S}{S^{*}} & =x_{1}, \frac{E}{E^{*}}=x_{2}, \frac{I_{s}}{I_{s}{ }^{*}}=x_{3}, \frac{I_{a}}{I_{a}{ }^{*}}=x_{4}, S^{*} I_{s}{ }^{*} \\
& =a, S^{*} I_{a}{ }^{*}=c, \beta_{s} b E^{*}=d, \beta_{a} b E^{*}=f
\end{aligned}
$$

and

$$
\begin{aligned}
& g\left(x_{1}, x_{2}, x_{3}, x_{4}\right)=\gamma K_{1}\left(a+c-a x_{1} x_{3}+c x_{1} x_{4}\right)-K_{1} \gamma a \frac{1}{x_{1}}-K_{1} \gamma c \frac{1}{x_{1}} \\
& +K_{1} \gamma a x_{3}+c K_{1} h x_{4}+K_{2} \gamma\left(a x_{1} x_{3}+c x_{1} x_{4}-a x_{2}-c x_{2}\right) \\
& -K_{2} \gamma\left(a x_{1} x_{3}\right) \frac{1}{x_{2}}-K_{2} \gamma\left(a x_{1} x_{4}\right) \frac{1}{x_{2}}+K_{2} \gamma a+K_{2} \gamma c+K_{3} d x_{2} \\
& -K_{3} d x_{3}-K_{3} d \frac{x_{2}}{x_{3}}+K_{3} d+K_{4}\left(f x_{2}-x_{4} f\right)-K_{4} f \frac{x_{2}}{x_{4}} \\
& +K_{4} f \\
& =\left(-K_{1} \gamma a+K_{2} \gamma a\right) x_{1} x_{3}+\left(-K_{1} \gamma c+K_{2} \gamma c\right) x_{1} x_{4}+\left(K_{1} \gamma a-K_{3} d\right) x_{3} \\
& \quad+x_{4}\left(K_{1} \gamma c-K_{4} f\right)+x_{2}\left(-K_{2} a+K_{4} f+K_{3} d\right)+K_{1} \gamma(a+c) \\
& \quad-K_{1} \gamma(a+c) \frac{1}{x_{1}}-K_{2} \gamma\left(a x_{1} x_{3}+c x_{1} x_{4}\right) \frac{1}{x_{2}}+K_{2} \gamma(a+c) \\
& \quad-K_{3} d \frac{x_{2}}{x_{3}}+K_{3} d-K_{4} f \frac{x_{2}}{x_{4}}+K_{4} f
\end{aligned}
$$

To get the values of $K_{1}, K_{2}, K_{3}, K_{4}$ we take the coefficients of $x_{1} x_{3}, x_{1} x_{4}, x_{4}, x_{3}, x_{2}$ equal to zero and solve the algebraic equations in $K_{1}, K_{2}, K_{3}, K_{4}$. This gives

$$
K_{1}=K_{2} ; K_{4}=\frac{K_{1} \gamma c}{f} ; K_{3}=\frac{K_{1} \gamma a}{d}
$$

Choosing $K_{1}=K_{2}=1$, we get

$$
\begin{aligned}
g\left(x_{1}, x_{2}, x_{3}, x_{4}\right)= & \gamma a\left(3-\frac{1}{x_{1}}-\frac{x_{1} x_{3}}{x_{2}}-\frac{x_{2}}{x_{3}}\right) \\
& +\gamma c\left(3-\frac{1}{x_{1}}-\frac{x_{1} x_{4}}{x_{2}}-\frac{x_{2}}{x_{4}}\right)
\end{aligned}
$$

Since the arithmetic mean (A.M.) is greater than or equal to geometric mean (G.M.), we have

$$
\frac{1}{x_{1}}+\frac{x_{1} x_{3}}{x_{2}}+\frac{x_{2}}{x_{3}} \geq 3 \text {, and } \frac{1}{x_{1}}+\frac{x_{1} x_{4}}{x_{2}}+\frac{x_{2}}{x_{4}} \geq 3 \text {. }
$$

Finally, we get

$$
\begin{aligned}
\frac{\mathrm{d} V_{1}}{\mathrm{~d} t}=-\frac{\left(S^{*}-S\right)^{2}}{S} \mu & +\gamma a\left(3-\frac{1}{x_{1}}-\frac{x_{1} x_{3}}{x_{2}}-\frac{x_{2}}{x_{3}}\right) \\
& +\gamma c\left(3-\frac{1}{x_{1}}-\frac{x_{1} x_{4}}{x_{2}}-\frac{x_{2}}{x_{4}}\right)
\end{aligned}
$$

Thus it is easy to observe that $\frac{\mathrm{d} V_{1}}{\mathrm{~d} t} \leq 0$ and the equality $\frac{\mathrm{d} V_{1}}{\mathrm{~d} t}=0$ hold only for $x_{1}=x_{2}=x_{3}=x_{4}=1$ for which $S=S^{*}, E=E^{*}, I_{s}=I_{s}{ }^{*}, I_{a}=I_{a}{ }^{*}$.

From the LaSalle's invariance principle (LaSalle 1976), the equilibrium $E_{1}$ of the given system is globally asymptotically stable for $R_{0}>1$.

\section{Numerical simulation}

Here numerical simulation is performed to support our analytical results. Most of our parameter values are from the reference Pongsumpun and Tang (2011), and the remaining parameters we have assumed. Here it is noted that all the parameters are in per day. The system (1) is simulated for different sets of parameters using MATLAB. In Fig. 2, we have shown stability of DFE $\left(E_{0}\right)$ using the following parameter values:

$$
\begin{aligned}
& \Lambda=3, \mu=0.00421, \beta_{s}=0.025, \beta_{a}=0.075, \\
& \gamma=0.0001, \delta=0.2, v=0.02, \psi=0.071, b=0.4 .
\end{aligned}
$$

For this set of parameters, the basic reproduction number $R_{0}=0.8179$ and the disease-free equilibrium point is (300, 0, 0, 0, 0, 0). In Figs. 3 and 4, we have shown stability of the Endemic equilibrium $\left(E_{1}\right)$ using the following set of parameters: 


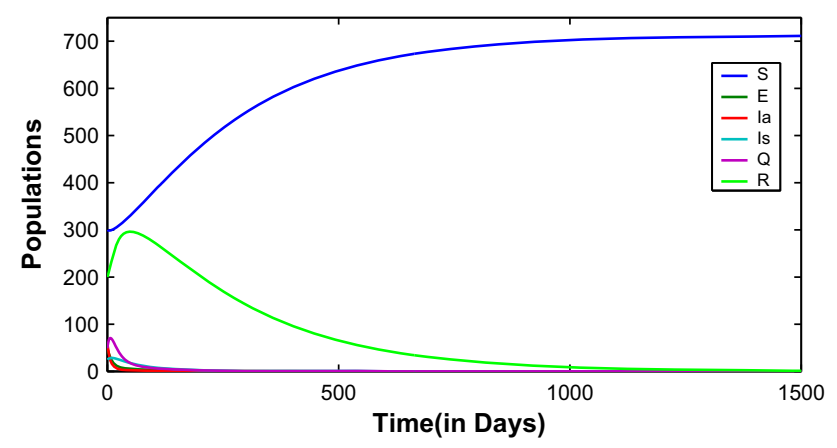

Fig. 2 Variation of $S E, I_{S}, I_{a}, Q, R$ showing the stability of disease free equilibrium point

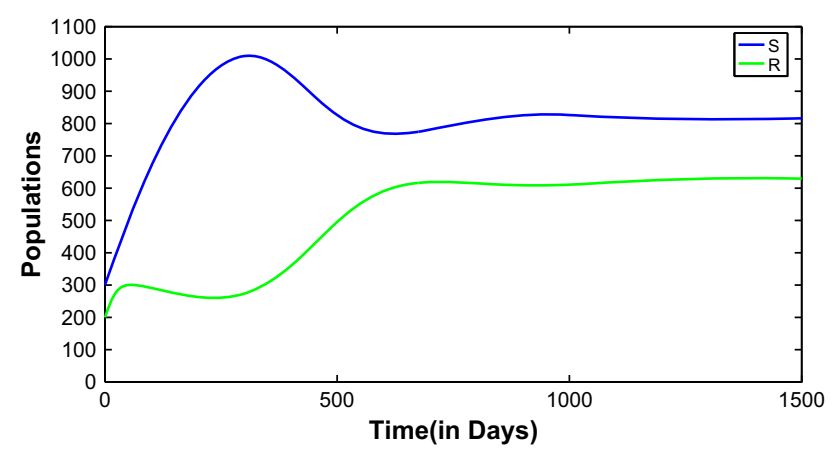

Fig. 3 Variation of $S$ and $R$ showing the stability of endemic equilibrium point

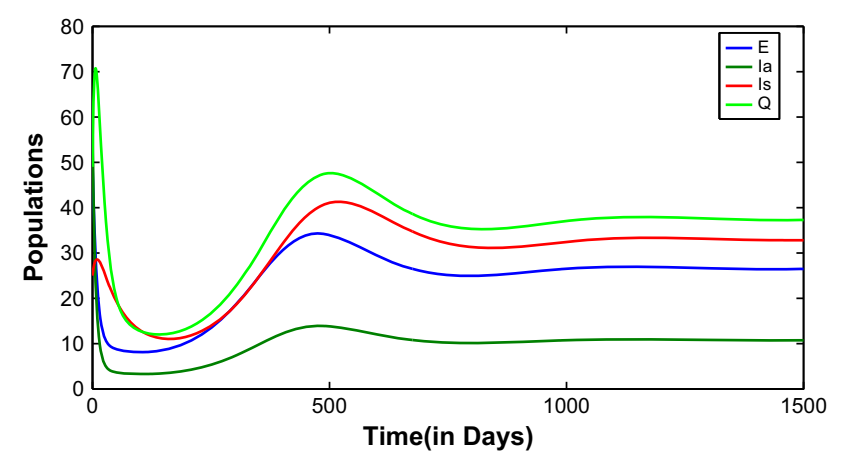

Fig. 4 Variation of $E, I_{s}, I_{a}$ and $Q$ showing the stability of endemic equilibrium point

$\Lambda=7, \mu=0.00421, \beta_{s}=0.025, \beta_{a}=0.075, \gamma=0.0001$,

$\delta=0.2, v=0.02, \psi=0.071, b=0.4, \eta=0.04227$.

For this parameters, the basic reproduction number $R_{0}=$ 2.0378 and the endemic equilibrium point is (354.43, $31.68, \quad 41.14, \quad 51.14, \quad 36.74, \quad 225.31)$. The effect of $\delta$ on equilibrium levels of $I_{a}$ and $I_{s}$, are demonstrated in the Figs. 5 and 6. It is observed that the equilibrium level of infective population decreases with the increase in $\delta$.

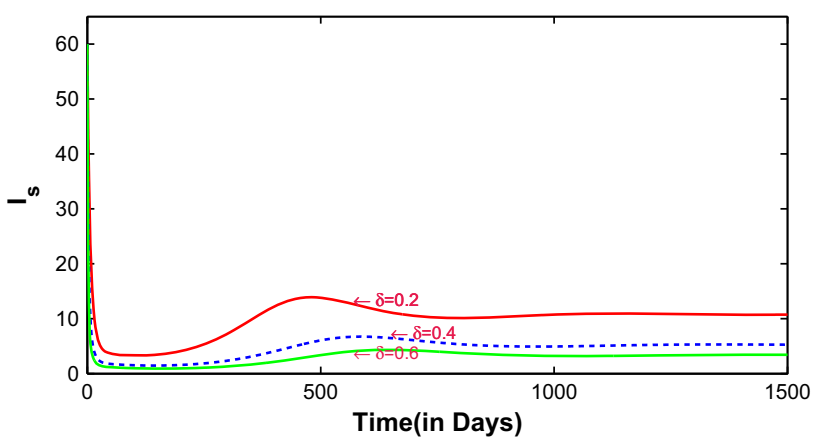

Fig. 5 Variation of $I_{s}$ with time showing the effect of $\delta$

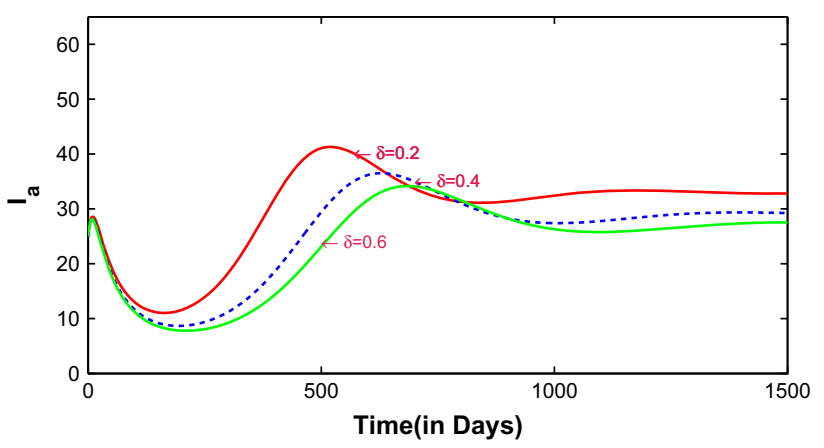

Fig. 6 Variation of $I_{a}$ with time showing the effect of $\delta$

\section{Optimal control problem}

Here we extend our model (1) by incorporating two optimal control parameters, namely, $u_{1}$ and $u_{2}$. The control $u_{1}(t)$ corresponds to the reduction in the transmission rate $(\gamma)$ and the control $u_{2}(t)$ corresponds to the increase in the rate of movement of symptomatic infectives to quarantine class. Our main aim is to minimize the transmission rate between susceptible individuals and infective individuals and also maximize the rate of quarantine of symptomatic infectives by adding additional time dependent rate $u_{2}(t)$ with minimal cost of controls. Both control functions are bounded and Lebesgue integrable on the interval $\left[0, t_{f}\right]$, where $t_{f}$ represents a pre-selected length of time during which these controls are applied. Our $u_{1}$ and $u_{2}$ must be equal to one for maximum control (effort). If $u_{1}$ and $u_{2}$ are equal to zero, then there is no effort being placed in these controls at time $t$. The optimal control system is given below:

$\frac{\mathrm{dS}}{\mathrm{d} t}=\Lambda-\mu S-\left(1-u_{1}(t)\right) \gamma I S$

$\frac{\mathrm{dE}}{\mathrm{d} t}=\left(1-u_{1}(t)\right) \gamma I S-\left(\beta_{s}+\beta_{a}\right) b E-\mu E$

$\frac{\mathrm{dI}_{\mathrm{s}}}{\mathrm{d} t}=\beta_{s} b E-\left(\delta+u_{2}(t)\right) I_{s}-\mu I_{s}-\eta I_{s}$ 
$\frac{\mathrm{dI}}{\mathrm{d} t}=\beta_{a} b E-v I_{a}-\mu I_{a}$

$\frac{\mathrm{dQ}}{\mathrm{d} t}=\left(\delta+u_{2}(t)\right) I_{s}+v I_{a}-\mu Q-\psi Q$

$\frac{\mathrm{dR}}{\mathrm{d} t}=\psi Q-\mu R$.

The objective functional for fixed duration of control $t_{f}$ is given below:

$J=\int_{0}^{t_{f}}\left(A_{1} I_{s}+A_{2} I_{a}+\frac{1}{2} c_{1} u_{1}^{2}+\frac{1}{2} c_{2} u_{2}^{2}\right) d t$,

where the parameter $A_{1} \geq 0, A_{2} \geq 0, c_{1} \geq 0, c_{2} \geq 0$, and it represent the weight constants.

Our objective is to find the control parameter $u_{1}{ }^{*}$ and $u_{2}{ }^{*}$, such that $J\left(u_{1}{ }^{*}, u_{2}{ }^{*}\right)=\min _{u_{1}, u_{2} \in \Omega} J\left(u_{1}, u_{2}\right)$, where $\Omega$ is the control set and is defined as $\Omega=\left\{u_{1}, u_{2}\right.$ : measurable and $0 \leq u_{1}, u_{2} \leq 1$ for $\left.t \in\left[0, t_{f}\right]\right\}$.

The Lagrangian of this problem is defined as:

$L\left(I_{s}, I_{a}, u_{1}, u_{2}\right)=A_{1} I_{s}+A_{2} I_{a}+\frac{1}{2} c_{1} u_{1}{ }^{2}+\frac{1}{2} c_{2} u_{2}{ }^{2}$

For our problem, we formed Hamiltonian $\mathcal{H}$ as follows:

$$
\begin{aligned}
\mathcal{H}=L\left(I_{s}, I_{a}, u_{1}, u_{2}\right) & +\lambda_{1} \frac{\mathrm{d} S}{\mathrm{~d} t}+\lambda_{2} \frac{\mathrm{d} E}{\mathrm{~d} t}+\lambda_{3} \frac{\mathrm{d} I_{s}}{\mathrm{~d} t} \\
& +\lambda_{4} \frac{\mathrm{d} I_{a}}{\mathrm{~d} t}+\lambda_{5} \frac{\mathrm{d} Q}{\mathrm{~d} t}+\lambda_{6} \frac{\mathrm{d} R}{\mathrm{~d} t},
\end{aligned}
$$

where $\lambda_{i}, i=1-6$ are the adjoint variables and are given by the solution of the following system of differential equations:

$$
\begin{aligned}
& \frac{\mathrm{d} \lambda_{1}}{\mathrm{~d} t}=-\frac{\partial \mathcal{H}}{\partial S}=\lambda_{1} \mu+\left(1-u_{1}(t)\right) \gamma I\left(\lambda_{1}-\lambda_{2}\right) \\
& \frac{\mathrm{d} \lambda_{2}}{\mathrm{~d} t}=-\frac{\partial \mathcal{H}}{\partial E}=\lambda_{2} \mu+\beta_{s} b\left(\lambda_{2}-\lambda_{3}\right)+\beta_{a} b\left(\lambda_{2}-\lambda_{4}\right) \\
& \frac{\mathrm{d} \lambda_{3}}{\mathrm{~d} t}=-\frac{\partial \mathcal{H}}{\partial I_{s}}=-A_{1}+\left(1-u_{1}(t)\right) \gamma S\left(\lambda_{1}-\lambda_{2}\right) \\
& +\left(\delta+u_{2}\right)\left(\lambda_{3}-\lambda_{5}\right)+\lambda_{3} \mu+\lambda_{3} \eta \\
& \frac{\mathrm{d} \lambda_{4}}{\mathrm{~d} t}=-\frac{\partial \mathcal{H}}{\partial I_{a}}=-A_{2}+\left(1-u_{1}(t)\right) \gamma S\left(\lambda_{1}-\lambda_{2}\right) \\
& +v\left(\lambda_{4}-\lambda_{5}\right)+\lambda_{4} \mu \\
& \frac{\mathrm{d} \lambda_{5}}{\mathrm{~d} t}=-\frac{\partial \mathcal{H}}{\partial Q}=\left(\lambda_{5}-\lambda_{6}\right) \psi+\lambda_{5} \mu \\
& \frac{\mathrm{d} \lambda_{6}}{\mathrm{~d} t}=-\frac{\partial \mathcal{H}}{\partial R}=\mu \lambda_{6}
\end{aligned}
$$$$
\text { satisfying the transversality }
$$$$
\lambda_{i}\left(t_{f}\right)=0, \text { for } i=1,2, \ldots 6 \text {. }
$$

condition
Let $\widetilde{S}, \widetilde{E}, \widetilde{I}_{s}, \widetilde{I}_{a}, \widetilde{Q}, \widetilde{R}$ be the optimum values of S, E, $I_{s}$, $I_{a}, Q, R$, and also let $\tilde{\lambda}_{1}, \tilde{\lambda}_{2}, \tilde{\lambda}_{3}, \tilde{\lambda}_{4}, \tilde{\lambda}_{5}, \tilde{\lambda}_{6}$, be the solution of the system. By using Pontryagin and Boltyanskii (1980) and Pontryagin et al. (1962) we state and prove the following theorem:

Theorem 4 There exist optimal controls $u_{1}{ }^{*}, u_{2}{ }^{*} \in \Omega$ such that $J\left(u_{1}{ }^{*}, u_{2}{ }^{*}\right)=\min _{u_{1}, u_{2} \in \Omega} J\left(u_{1}, u_{2}\right)$ subject to system (3).

Proof To prove this theorem we use Pontryagin et al. (1962). Here the state variable and the controls are positive. For this minimizing problem, the necessary convexity of the objective functional in $\left(u_{1}, u_{2}\right)$ is satisfied. The control variable set $\Omega$, where $u_{1}, u_{2} \in \Omega$ is also convex and closed by the definition. The integrand of the functional $\left(A_{1} I_{s}+A_{2} I_{a}+\frac{1}{2} c_{1} u_{1}^{2}+\frac{1}{2} c_{2} u_{2}^{2}\right)$ is convex on the control set $\Omega$ and the state variables are bounded.

Since there exists an optimal control for minimizing the functional subject to given mathematical model and adjoint variables. To derive necessary condition and to find optimal solution, we use Pontryagin's maximum principle.

If $(x, u)$ is an optimal solution of an optimal control problem, then there exist a non-trivial vector function $\lambda=$ $\lambda_{1}, \lambda_{2}, \lambda_{3}, \ldots, \lambda_{n}$ satisfying the following inequalities.

$$
\begin{aligned}
\frac{\mathrm{d} x}{\mathrm{~d} t} & =\frac{\partial H(t, x, u, \lambda)}{\partial \lambda} \\
0 & =\frac{\partial H(t, x, u, \lambda)}{\partial \lambda} \\
\frac{\mathrm{d} \lambda}{\mathrm{d} t} & =\frac{\partial H(t, x, u, \lambda)}{\partial \lambda}
\end{aligned}
$$

With the help of Pontryagin's maximum principle (Pontryagin and Boltyanskii 1980) we proved the following theorem:

Theorem 5 The optimal controls $\left(u_{1}{ }^{*}, u_{2}{ }^{*}\right)$ which minimize $J$ over the region $\Omega$ are given by

$$
\begin{aligned}
& u_{1}{ }^{*}=\min \left\{1, \max \left(0, \widetilde{u_{1}}\right)\right\} \\
& u_{2}{ }^{*}=\min \left\{1, \max \left(0, \widetilde{u_{2}}\right)\right\}
\end{aligned}
$$

where

$$
\begin{aligned}
& \widetilde{u_{1}}=\frac{\gamma\left(\widetilde{I_{a}}+\widetilde{I_{s}}\right) \widetilde{S}\left(\lambda_{2}-\lambda_{1}\right)}{c_{1}} \\
& \widetilde{u_{2}}=\frac{\left(\lambda_{3}-\lambda_{5}\right) \widetilde{I}_{s}}{c_{2}}
\end{aligned}
$$

Proof Using optimally condition:

$\frac{\partial \mathcal{H}}{\partial u_{1}}=0, \quad \frac{\partial \mathcal{H}}{\partial u_{2}}=0$, 
$u_{1}=\frac{\left(\lambda_{2}-\lambda_{1}\right) \gamma \widetilde{S} \widetilde{I}}{c_{1}}=\widetilde{u_{1}} \quad$ and,$\quad u_{2}=\frac{\left(\lambda_{3}-\lambda_{5}\right) \widetilde{I}_{s}}{c_{2}}=\widetilde{u_{2}}$

Again upper and lower bounds for these control are 0 and 1 respectively. i.e. $u_{1}=u_{2}=0$ if $\widetilde{u_{1}}<0$ and $\widetilde{u_{2}}<0$, and $u_{1}=u_{2}=1$ if $\tilde{u_{1}}>1$ and $\widetilde{u_{2}}>1$, otherwise $u_{1}=\widetilde{u_{1}}$ and $u_{2}=\widetilde{u_{2}}$. Hence for these controls $u_{1}{ }^{*}, u_{2}{ }^{*}$ we get optimum value of the function $J$. This completes the proof of the theorem.

\section{Simulation of the optimal control model}

The following set of parameters are used to simulate the optimal control model. Here it is noted that most of the parameters used here are same as the parameter values used to get endemic equilibrium for the model (1).

$\Lambda=7, \mu=0.00421, \beta_{s}=0.025, \beta_{a}=0.075, \gamma=0.0001$,

$\delta=0.2, v=0.02, \psi=0.071, b=0.4, \eta=0.04227$.

The time interval for which optimal control is applied is taken as 300 days. At first we solve the state equations by the forward Euler method in the time interval [0, 300] starting with an initial guess for the control. Then we solve the adjoint system using the solutions of the state system and the transversality conditions backward in time. The control profile of $u_{1}(t)$ and $u_{2}(t)$ are shown in Figs. 7 and 8 . Variation of the symptomatic infective population with and without optimal control is demonstrated in Fig. 9. From this figure, one can see the significant decrease in the infective population in presence of optimal control. From Figs. 7 and 8, it is observed that the control $u_{2}(t)$ is required more compared to the control $u_{1}(t)$. So in Figs. 10 and 11, we have tried to see the effect of different values of weight constant $c_{2}$ on the control profile $u_{2}(t)$ and the corresponding effect on the symptomatic infective population $I_{s}$. From these figures it is observed that when we increase the weight constant $C_{2}$, the

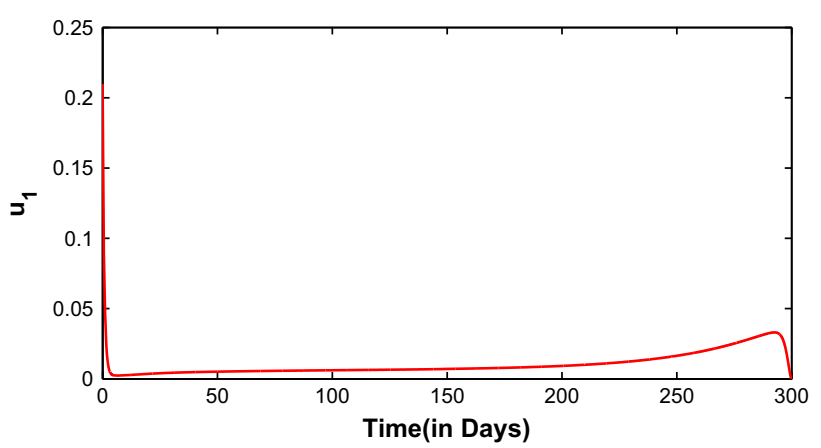

Fig. 7 Control profile of $u_{1}$

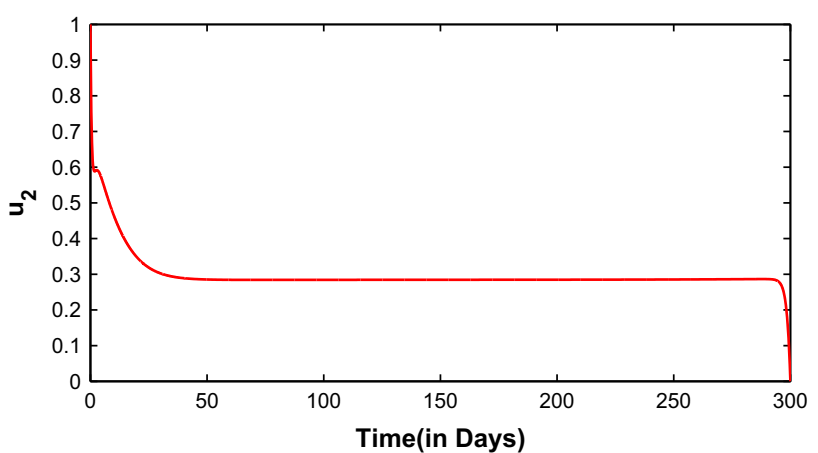

Fig. 8 Control profile of $u_{2}$

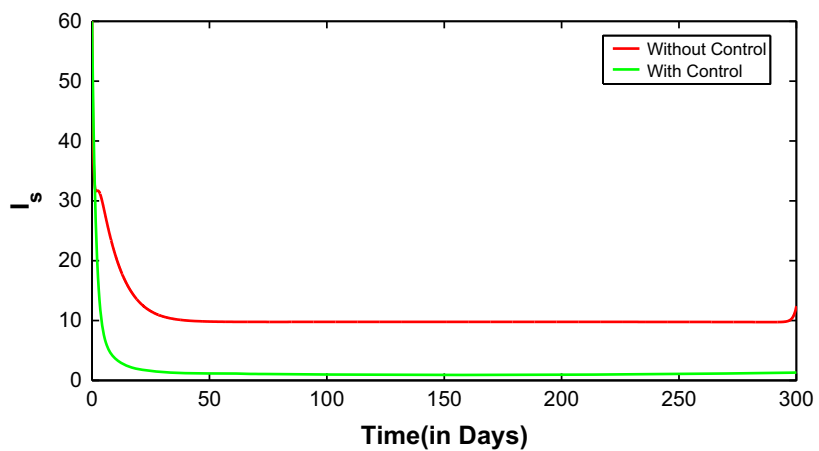

Fig. 9 Variation of $I_{s}$ with and without optimal control

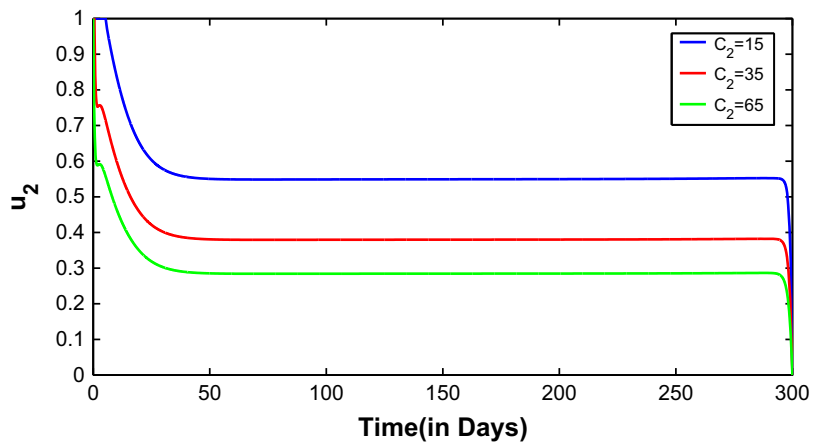

Fig. 10 Optimal control profile of $u_{2}$ for different costs of control $C_{2}$

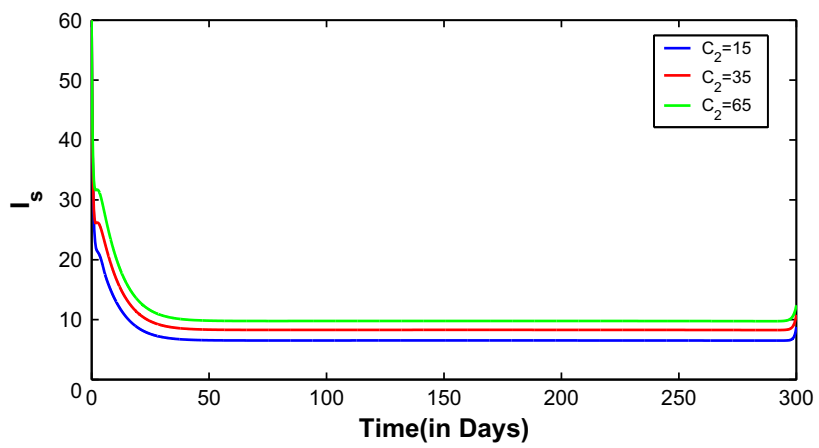

Fig. 11 Variation of $I_{s}$ for different costs of control $C_{2}$ 
cost of efforts increases and optimal control decreases, leading to increase in the number of infectives due to reduction in the intensity of intervention.

\section{Conclusion}

An epidemic model for transmission dynamics of Swine flu disease is proposed and analyzed. There exist two equilibria, namely the disease-free equilibrium (DFE) and the endemic equilibrium (EP). The DFE is globally asymptotically stable whenever the basic reproduction number $R_{0}$ is less than unity. The endemic equilibrium (EE) is also globally asymptotically stable whenever it exists. Numerical simulation is performed to support our analytical results. Also it supports the fact that increase in the parameter $\delta$ (rate of quarantine) causes the decrease in the equilibrium level of the infective population. Finally, we extend our model to optimal control problem and analyze it. Numerical simulation is extended to this model too to see the effect of optimal controls. The symptomatic infectives is plotted against time with and without optimal control. The control profile for both the controls are obtained and it is observed that optimal control gives better result in reducing the number of infectives in desired time interval. The effect of weight constant $C_{2}$ associated with the optimal control parameter $u_{2}$ on the infectives are also studied. Here it is noted that $u_{2}(t)$ is the additional time dependent rate of quarantine and increase in the cost of quarantine leads to increase in the number of infectives.

\section{References}

MHFW (2016) Swine Flu-H1N1 (Seasonal Influenza), Ministry of Health \& Family Welfare, India. http://mohfw.gov.in/index4. php?lang $=1 \&$ level $=0 \&$ linkid $=372 \&$ lid $=3066$. Accessed $24 \mathrm{Feb}$ 2016

CDC (2016) Flu symptoms \& severity, centre for disease control and prevention. http://www.cdc.gov/flu/consumer/symptoms.htm. Accessed 10 Jan 2016
Sharomi O, Podder CN, Gumel AB, Mahmud SM, Rubinstein E (2011) Modelling the transmission dynamics and control of the novel 2009 swine influenza (H1N1) pandemic. Bull Math Biol 73:515-548

Gonzlez-Parra G, Arenas Abraham J, Aranda Diego F, Segovia L (2011) Modeling the epidemic waves of AH1N1/09 influenza around the world. Spat Spat Temp Epidemiol 2(4):219-226

Changpuek T, Pongsumpun P, Tang I (2013) Analysis of mathematical model for swine flu transmission by age group. Far East $\mathrm{J}$ Math Sci 73(2):201-229

Aldila D, Nuraini N, Soewono E (2014) Optimal control problem in preventing of swine flu disease transmission. Appl Math Sci 8(71):3501-3512

Pongsumpun P, Tang IM (2011) Mathematical model of the symptomatic and asymptomatic infections of swine flu. Int $\mathbf{J}$ Math Models Methods Appl Sci 2(4):247-254

Lakshmikantham V, Leela S, Martynyuk AA (1989) Stability analysis of nonlinear systems. Marcel Dekker Inc., New York

Centers for Disease Control and Prevention (2009d) Outbreak of swine-origin influenza A (H1N1) virus infection-Mexico. Morb Mort Wkly Rep 58:13

World Health Organization (2009) Human infection with new influenza A (H1N1) virus: clinical observations from Mexico and other affected countries. Wkly Epidemiol Rec 84:185

Centers for Disease Control and Prevention (2009a) http://www.cdc. gov/h1n1flu/background.htm. Accessed 10 Jan 2016

Centers for Disease Control and Prevention (2009b). http://www.cdc. gov/h1n1flu/recommendations.htm. Accessed 10 Jan 2016

Centers for Disease Control and Prevention (2009c). http://www.cdc. gov/media/pressrel/2009/r090729b.htm. Accessed 10 Jan 2016

Korobeinikov A, Wake GC (2002) Lyapunov function and global stability for SIR, SIRS, and SIS epidemiological models. Appl. Math. Lett. 15:955-960

Li MY, Muldowney JS (1995) Global stability for the SEIR model in epidemiology. Math Biosci 125:155-164

Li MY, Muldowney JS (1996) A geometric approach to global stability problems. SIAM J Math Anal 27(4):1070-1083

LaSalle JP (1976) The stability of dynamical systems. Regional conference series in applied mathematics. SIAM, Philadelphia

Pontryagin LS, Boltyanskii VG (1980) The mathematical theory of optimal processes. Golden and Breach Science Publishers, London

Pontryagin LS, Boltyanskii VG, Gamkrelidze RV, Mischenko EF (1962) The mathematical theory of optimal process. Wiley, New York

Lenhart S, Workman J (2007) Optimal control applied to biological model. Chapman \& Hall/CRC, London 